\title{
Ajustamento para heterogeneidade de variâncias para produção de leite e gordura entre rebanhos da raça Pardo-Suíça no Brasil
}

[Adjustment for heterogeneity of variance for milk and fat yield among herds of Brown Swiss in Brazil]

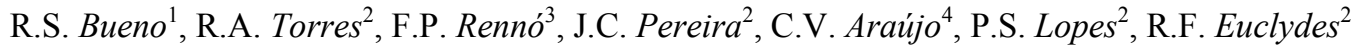 \\ ${ }^{1}$ Aluna de pós-graduação - UFV - Viçosa, MG \\ ${ }^{2}$ Departamento de Zootecnia - UFV - Viçosa, MG \\ ${ }^{3}$ Faculdade de Medicina Veterinária e Zootecnia - USP - São Paulo, SP \\ ${ }^{4}$ Universidade Federal Rural da Amazônia - Belém, PA
}

\begin{abstract}
RESUMO
Para verificar o efeito da inclusão das interações reprodutor $\mathrm{x}$ rebanho e reprodutor $\mathrm{x}$ rebanho-ano como fator de ajustamento da heterogeneidade de variância, registros de produção de leite e gordura foram estratificados, com base no desvio-padrão fenotípico da produção de leite ajustada, em duas classes: baixo $(\leq 1.280 \mathrm{~kg})$ e alto $(>1.280 \mathrm{~kg})$ desvio-padrão. Três modelos foram utilizados, sem e com interação reprodutor x rebanho e reprodutor $\mathrm{x}$ rebanho-ano, em análises de característica única, geral e em cada classe de desvio-padrão. Médias e componentes de variâncias foram maiores na classe de alto desviopadrão. Na classe de baixo desvio-padrão, a herdabilidade não se alterou com a inclusão dos efeitos de interação no modelo, sendo de 0,34 para produção de leite e de 0,32 para produção de gordura. Na classe de alto desvio-padrão, as herdabilidades foram: $0,37,0,35$ e 0,36 , e de $0,35,0,32$ e 0,35 , para produção de leite e gordura, nos modelos sem, com interação reprodutor $\mathrm{x}$ rebanho e com interação reprodutor $\mathrm{x}$ rebanho-ano, respectivamente. A inclusão do efeito de interação reprodutor x rebanho nos modelos foi significativa $(\mathrm{P}<0,01)$ para produção de gordura, em análise geral e na classe de alto desvio-padrão, pelo teste da razão de verossimilhança.
\end{abstract}

Palavras-chave: gado de leite, produção de leite, produção de gordura, componentes de variância, interação reprodutor $\mathrm{x}$ rebanho, parâmetros genéticos

\begin{abstract}
In order to verify the effect of including the interactions of sire $x$ herd and the sire $x$ herd-year, as a adjust factor of the variance heterogeneity, registers of milk and fat yields were classified into two classes of standard deviation: low $(\leq 1.280 \mathrm{~kg})$ and high $(>1.280 \mathrm{~kg})$, based on phenotypic standard deviation of the milk production adjusted. Three models, without and with interaction of sire $x$ herd and sire $x$ herdyear, were used in the general univariate analyses and in each standard deviation class. Averages and variance components were higher in the high standard deviation. In the class of low standard deviation, heritability didn't alter with the inclusion of the interaction effects in the model, being of 0.34 for milk yield and 0.32 for fat yield. In the class of high standard deviation, heritabilities were: 0.37, 0.35 and 0.36 , and of 0.35, 0.32 and 0.35 , for milk and fat yield, in the models without and with interaction of sire $x$ herd and with interaction of sire $x$ herd-year, respectively. The inclusion of the interaction of sire $x$ herd was significant $(P<0.01)$ for fat yield, in general analyses and in the high standard deviation class, in the likelihood ratio test.
\end{abstract}

Keywords: dairy cattle, milk yield, fat yield, variance components, sire $x$ herd interaction, genetic parameters

Recebido em 25 de abril de 2005

Aceito em 21 de dezembro de 2006

E-mail: rachelbueno@hotmail.com 


\section{INTRODUÇÃO}

Geralmente, num sistema de avaliação genética, assume-se homogeneidade das variâncias genética e residual para todos os ambientes. Esta pressuposição pode não ser verdadeira quando as condições ambientais forem heterogêneas ou quando os desempenhos são obtidos durante um longo período de tempo.

As avaliações genéticas em gado de leite são comumente realizadas usando-se dados de produção de vários rebanhos, abrangendo informações de todo território nacional. Por esta razão, os rebanhos chegam a diferir muito em relação à média de produção e à variância fenotípica. Sob essas condições, até mesmo os procedimentos de avaliação genética que fornecem soluções com propriedades de melhor predição linear não-viesada (BLUP), estão sujeitos a erros, podendo comprometer o progresso genético esperado pela seleção, quando realizada entre rebanhos.

Variâncias heterogêneas entre rebanhos e aumento da variância, de acordo com o aumento dos níveis de produções dos rebanhos, têm sido verificados por vários autores, como De Veer e Van Vleck (1987), Boldman e Freeman (1990), Dong e Mao (1990), Torres (1998) e Araújo (2000). Dentre as fontes de variância heterogênea, provavelmente a mais importante seja o aumento da variância fenotípica com a melhoria do nível de produção como um efeito de escala.

$\mathrm{Na}$ avaliação genética de reprodutores, é importante considerar a variabilidade dentro de níveis de produção, pois, sob seleção, as classes de maior variância contribuem com maior parte dos animais, e a avaliação genética do animal pode ser mais em função do ambiente do que do seu potencial genético (Torres, 1998). A explicação para tal fato é que melhores condições de manejo possibilita melhor expressão do verdadeiro potencial genético dos animais (Vinson, 1987). Assim, torna-se necessário conhecer as formas de ajustes que podem ser utilizadas para estabilizar as variâncias e se considerar as diferenças de herdabilidade para produção de leite nos rebanhos ao se fazerem avaliações.
O objetivo deste trabalho foi verificar o efeito da inclusão da interação reprodutor $\mathrm{x}$ rebanho $\mathrm{e}$ reprodutor x rebanho-ano no modelo de análise, como fator de ajustamento para variâncias heterogêneas, bem como, verificar o impacto de heterogeneidade de variâncias nas estimativas dos componentes de variância e herdabilidade.

\section{MATERIAL E MÉTODOS}

O conjunto inicial de dados era constituído de 12.066 registros de lactações de 5.798 vacas distribuídas em 269 rebanhos, com parições nos anos de 1980 a 2002. Essas informações, provenientes do Controle Leiteiro da Associação Brasileira dos Criadores de Gado Pardo-Suíço (ABCGPS), foram fornecidas pelo convênio mantido entre o Departamento de Zootecnia da Universidade Federal de Viçosa e a ABCGPS.

Os dados foram editados para eliminação de registros de produção e ascendência incompleta, lactações encerradas por causas anormais de secagem, lactações com duração inferior a 150 e superior a 450 dias, registros de produção de leite na lactação inferiores a $1.000 \mathrm{~kg}$ ou superiores a $15.000 \mathrm{~kg}$ e, ainda, idade da vaca ao parto menor que 18 e maior que 197 meses; foram considerados somente os registros das quatro primeiras lactações.

Quatro estações de parto foram definidas: estação 1, que corresponde aos meses de janeiro a março; estação 2, que corresponde aos meses de abril a junho; estação 3, correspondendo aos meses de julho a setembro; e estação 4, que corresponde aos meses de outubro a dezembro. As estações 1 e 4 correspondem aos meses de maior incidência de chuvas e de temperaturas e umidade relativa do ar mais elevadas, enquanto que as estações 2 e 3 correspondem aos meses de menor incidência de chuvas e de temperaturas e umidade relativa do ar mais baixas, já que a maior parte dos dados corresponde a rebanhos localizados nas regiões sudeste e sul do país.

As vacas foram classificadas em três grupos genéticos: grupo 1, formado por vacas PardoSuíças puras de origem (PO), grupo 2, vacas Pardo-Suíças puras por cruzamento (PC) e grupo 3, vacas Pardo-Suíças puras de origem importadas (POI). Posteriormente, os efeitos fixos de ano e rebanho foram agrupados em classes. 
Os registros de produção foram previamente ajustados para número de ordenhas (duas ordenhas), duração da lactação (305 dias) e para produção à idade adulta da vaca (72 e 69 meses, para produção de leite e gordura, respectivamente) utilizando fatores estimados por Rennó (2001).

Com o intuito de avaliar o efeito da heterogeneidade de variâncias sobre a produção de leite e de gordura, utilizou-se o desvio-padrão fenotípico dos rebanhos para produção de leite ajustada, como critério para estratificar os dados em duas classes, com número aproximado de observações nas duas classes. A classe de baixo desvio-padrão fenotípico corresponde aos dados cujo desvio-padrão de rebanho foram menores ou iguais a $1.280 \mathrm{~kg}$, e a de alto desvio-padrão fenotípico, maiores que $1.280 \mathrm{~kg}$. Depois de formadas as classes, impôs-se a restrição de que cada classe de rebanho-ano de parto deveria apresentar, no mínimo, quatro observações e que cada reprodutor deveria ter, no mínimo, quatro filhas, estar presente nas duas classes de desviopadrão, e possuir filhas em dois diferentes rebanhos dentro de cada classe. Após imposição das restrições, 7.046 lactações de 3.569 vacas, filhas de 70 reprodutores foram obtidas.

A matriz de numeradores dos coeficientes de parentesco (NRM), utilizada em todas as análises, foi obtida de 8.682 animais diferentes, entre os quais 17 eram endogâmicos, o que levou a um coeficiente de endogamia médio de 0,14 .

As análises foram conduzidas utilizando-se três modelos: 1) modelo padrão, com ausência do efeito da interação, 2) modelo padrão com a inclusão da interação reprodutor $\mathrm{x}$ rebanho, e 3 ) modelo padrão com a interação reprodutor $\mathrm{x}$ rebanho-ano. Foram realizadas análises de característica única com os dados de produção de leite e de gordura do leite, ajustada a duas ordenhas diárias, a 305 dias de lactação e a idade adulta da vaca, dentro de cada classe e em análise geral, desconsiderando a formação das classes de desvio-padrão fenotípico.

Em notação matricial, o modelo estatístico utilizado, que considera o efeito da interação reprodutor $x$ rebanho-ano, é:

$y=X \beta+Z_{a} a+Z_{p} p+Z_{\text {sxhxy }} t+e$, em que: $\mathrm{y}=$ vetor, $\mathrm{n} \times 1$, de $\mathrm{n}$ observações da característica produção de leite ou produção de gordura; $X=$ matriz, $\mathrm{n} \times \mathrm{f}$, de incidência de níveis dos efeitos fixos; $\beta=$ vetor, $\mathrm{f} \times 1$, de efeitos fixos; $Z_{\mathrm{a}}=$ matriz, $\mathrm{n} \times \mathrm{N}$, de incidência dos valores genéticos; $\mathrm{a}=$ vetor, $\mathrm{N} \times 1$, de valores genéticos dos animais; $Z_{\mathrm{p}}=$ matriz, $\mathrm{n} \times$ $\mathrm{N}$, de incidência dos efeitos de ambiente permanente sobre os animais; $\mathrm{p}=$ vetor, $\mathrm{N} \times 1$, de valores referentes ao efeito de ambiente permanente dos animais; $Z_{\text {sxhxy }}=$ matriz, $\mathrm{nx} \mathrm{v}$, de incidência dos efeitos da interação reprodutor $\mathrm{x}$ rebanho-ano; $\mathrm{t}=$ vetor, $\mathrm{v} \times 1$, de valores referentes ao efeito da interação reprodutor $\mathrm{x}$ rebanho-ano; e e = vetor de resíduos da mesma dimensão de $\mathrm{y}$; sendo: $\mathrm{N}=$ número de indivíduos; $\mathrm{n}=$ número total de observações; $\mathrm{f}=$ número de classes de efeitos fixos; e $\mathrm{v}=$ número de níveis da interação reprodutor $\mathrm{x}$ rebanho-ano.

As pressuposições acerca das distribuições dos fatores, de efeito aleatório, contidos no modelo estatístico, e as equações de modelo misto são representadas da mesma forma como descrito por Araújo (2000). Nas análises que consideram o efeito da interação reprodutor $\mathrm{x}$ rebanho, $\mathrm{o}$ modelo é semelhante ao descrito anteriormente, com a inclusão da matriz $Z_{\text {sxh }}$ e do vetor $s$, em substituição ao componente da interação reprodutor $\mathrm{x}$ rebanho-ano, em que: $\mathrm{Z}_{\mathrm{sxh}}=$ matriz de incidência de dimensão $\mathrm{n} x \mathrm{~h}$, referente aos efeitos da interação reprodutor x rebanho; e $\mathrm{s}=$ vetor, $\mathrm{h} \times 1$, ou seja, igual ao número de níveis de interação reprodutor $\mathrm{x}$ rebanho. As análises sem inclusão do efeito de interação diferem destas apenas pela ausência dos componentes desses efeitos nos modelos.

A estimação de componentes de (co)variâncias e parâmetros genéticos foram feitas pelo uso do programa Multiple Trait Derivative Free Restricted Maximum Likelihood (MTDFREML), segundo Boldman et al. (1995). Adotou-se como critério de convergência a variância dos valores da função $-2 \log \lambda$, do 'simplex', como sendo menor que $10^{-9}$.

Para determinar a importância da inclusão do efeito da interação reprodutor $\mathrm{x}$ rebanho ou reprodutor x rebanho-ano no modelo, utilizou-se 
o teste da razão de verossimilhança (LR) para modelos seqüencialmente reduzidos (Rao, 1973). A estatística LR foi comparada com os valores da distribuição de qui-quadrado ( $\chi^{2}$ tabelado), com um grau de liberdade e nível de significância fixo de $1 \%$.

\section{RESULTADOS E DISCUSSÃO}

As médias observadas, os desvios-padrão, os coeficientes de variação e o número de lactações, para produção de leite e de gordura do leite, ajustada a duas ordenhas diárias, a 305 dias de lactação e à idade adulta da vaca, em cada classe de desvio-padrão fenotípico e em análise geral, são apresentadas na Tab. 1. Os componentes de variância, para cada classe de desvio-padrão fenotípico e em análise geral, estimados pelos modelos sem, com interação reprodutor $\mathrm{x}$ rebanho e com interação reprodutor $\mathrm{x}$ rebanhoano, podem ser observados na Tab. 2 .
As médias da produção de leite e de gordura aumentaram da classe de baixo desvio-padrão fenotípico para a classe de alto desvio-padrão fenotípico confirmando a associação entre média e desvio-padrão dentro de rebanho.

Os componentes de variância, para produção de leite e de gordura, referentes ao efeito genético aditivo, ao efeito de ambiente permanente, ao efeito da interação reprodutor $\mathrm{x}$ rebanho $\mathrm{e}$ reprodutor $\mathrm{x}$ rebanho-ano, quando considerados no modelo, e ao efeito residual, aumentaram da classe de baixo desvio-padrão fenotípico para a classe de alto desvio-padrão fenotípico. Aumento nas estimativas de componentes de variância genética e residual, à medida que o nível de produção dos rebanhos aumenta, foi também observado por Hill et al. (1983), Torres (1998) e Araújo (2000).

Tabela 1. Médias \pm desvios-padrão, coeficientes de variação $(\mathrm{CV})$ e número de lactações para as características produção de leite e de gordura do leite, ajustada a 305 dias de lactação, a duas ordenhas diárias e à idade adulta, em cada classe de desvio-padrão e em análise geral

\begin{tabular}{|c|c|c|c|c|}
\hline Desvio-padrão & Média $(\mathrm{kg})$ & Desvio-padrão (kg) & CV $(\%)$ & $\mathrm{N}^{\circ}$ de lactações \\
\hline \multicolumn{5}{|c|}{ Produção de leite } \\
\hline Geral $^{1}$ & $6.142,04$ & $1.716,41$ & 27,94 & 7.046 \\
\hline Alto & $6.710,88$ & $1.734,95$ & 25,85 & 3.768 \\
\hline Baixo & $5.488,16$ & $1.440,02$ & 26,24 & 3.278 \\
\hline \multicolumn{5}{|c|}{ Produção de gordura } \\
\hline Geral $^{1}$ & 229,34 & 64,41 & 28,09 & 7.046 \\
\hline Alto & 249,12 & 66,19 & 26,58 & 3.768 \\
\hline Baixo & 206,61 & 54,00 & 26,14 & 3.278 \\
\hline
\end{tabular}

${ }^{1}$ Inclui as classes de alto e baixo desvio-padrão fenotípico

As estimativas de componentes de variância pouco alteraram com a inclusão nos modelos dos efeitos de interação. Quando o modelo considerava o efeito $\mathrm{da}$ interação reprodutor $\mathrm{x}$ rebanho, ocorreram reduções de 3,5 e 8,6\%, na variância genética aditiva, e de 3,1 e $8,3 \%$, na variância de ambiente permanente, respectivamente, para produção de leite e de gordura, na classe de alto desvio-padrão fenotípico. No modelo com interação reprodutor $\mathrm{x}$ rebanho-ano, os componentes de variância residual reduziram em 3,0 e 2,8\% para produção de leite, e para produção de gordura em 2,6 e $1,9 \%$, nas classes de baixo e alto desvio-padrão fenotípico, respectivamente.

Reduções nas estimativas de componentes de variância foram também observadas por Araújo (2000) ao comparar modelos com e sem efeito de interação reprodutor $\mathrm{x}$ rebanho. Os resultados desse estudo indicam que os componentes de variância genética aditiva reduziram em 8,2 e $7 \%$, nas classes de alto, médio e baixo desvio-padrão fenotípico, respectivamente. As reduções nos componentes de efeito permanente de meio foram de 12,8 e $6 \%$, nas classes de alto, médio e baixo desvio-padrão fenotípico, respectivamente. Para os componentes de variâncias residuais, a redução foi menor que $1 \%$ em todas as classes de desvio-padrão fenotípicos.

$\mathrm{Na}$ Tab. 3 são apresentadas as estimativas de herdabilidade e as proporções da variância total, referentes aos efeitos de ambiente permanente, de interação reprodutor $\mathrm{x}$ rebanho $\mathrm{e}$ reprodutor $\mathrm{x}$ rebanho-ano, e residual, em análise geral, e em cada classe de desvio-padrão fenotípico. 
Tabela 2. Estimativas de componentes de variância genética aditiva $\left(\hat{\sigma}_{\mathrm{a}}^{2}\right)$, ambiente permanente $\left(\hat{\sigma}_{\mathrm{C}^{1}}^{2}\right)$, interação reprodutor x rebanho $\left(\hat{\sigma}_{C^{2}}^{2}\right)$, interação reprodutor x rebanho-ano $\left(\hat{\sigma}_{C^{3}}^{2}\right)$ e residual $\left(\hat{\sigma}_{e}^{2}\right)$ para produção de leite e gordura, em análise geral e em cada classe de desvio-padrão

\begin{tabular}{|c|c|c|c|c|c|c|}
\hline \multirow{2}{*}{$\begin{array}{l}\text { Componente } \\
\text { de variância }\end{array}$} & \multicolumn{3}{|c|}{ Produção de leite } & \multicolumn{3}{|c|}{ Produção de gordura } \\
\hline & Baixo & Alto & Geral & Baixo & Alto & Geral \\
\hline & \multicolumn{6}{|c|}{ Modelo sem interação } \\
\hline$\hat{\sigma}_{\mathrm{a}}^{2}$ & $346.433,33$ & $636.274,82$ & $508.197,09$ & 473,08 & 899,12 & 705,93 \\
\hline$\hat{\sigma}_{\mathrm{C}^{1}}^{2}$ & $23.292,99$ & $53.617,53$ & $39.519,28$ & 26,28 & 78,04 & 54,50 \\
\hline \multirow[t]{2}{*}{$\hat{\sigma}_{\mathrm{e}}^{2}$} & $643.556,25$ & $1.049 .361,34$ & $859.472,02$ & 979,63 & $1.615,36$ & $1.318,85$ \\
\hline & \multicolumn{6}{|c|}{ Modelo com interação reprodutor x rebanho } \\
\hline$\hat{\sigma}_{\mathrm{a}}^{2}$ & $346.433,41$ & $614.139,38$ & $498.363,69$ & 472,46 & 822,11 & 655,84 \\
\hline$\hat{\sigma}_{C^{1}}^{2}$ & $23.286,90$ & $51.942,03$ & $38.562,52$ & 26,26 & 71,55 & 49,61 \\
\hline$\hat{\sigma}_{\mathrm{C}^{2}}^{2}$ & 1,87 & $27.542,04$ & $13.540,64$ & 0,84 & 97,62 & 67,89 \\
\hline \multirow[t]{2}{*}{$\hat{\sigma}_{\mathrm{e}}^{2}$} & $643.568,29$ & $1.047 .916,77$ & $858.921,34$ & 979,66 & $1.611,13$ & $1.316,45$ \\
\hline & \multicolumn{6}{|c|}{ Modelo com interação reprodutor $\mathrm{x}$ rebanho-ano } \\
\hline$\hat{\sigma}_{\mathrm{a}}^{2}$ & $346.918,55$ & $633.424,30$ & $506.124,03$ & 470,85 & 894,75 & 699,84 \\
\hline$\hat{\sigma}_{C^{1}}^{2}$ & $23.287,93$ & $53.577,89$ & $39.399,16$ & 26,42 & 77,83 & 53,99 \\
\hline$\hat{\sigma}_{C^{3}}^{2}$ & $23.406,06$ & $33.902,36$ & $38.290,04$ & 32,45 & 37,05 & 56,53 \\
\hline$\hat{\sigma}_{\mathrm{e}}^{2}$ & $624.085,73$ & $1.019 .726,18$ & $827.318,16$ & 953,70 & $1.584,51$ & $1.273,63$ \\
\hline
\end{tabular}

Tabela 3. Estimativas de herdabilidade e proporções da variância fenotípica em relação aos efeitos de ambiente permanente $\left(\mathrm{C}^{1}\right)$, efeito da interação reprodutor $\mathrm{x}$ rebanho $\left(\mathrm{C}^{2}\right)$ e reprodutor $\mathrm{x}$ rebanho-ano $\left(\mathrm{C}^{3}\right)$ quando consideradas no modelo, e efeito residual (e) para as características produção de leite e gordura, em análise geral e em cada classe de desvio-padrão

\begin{tabular}{|c|c|c|c|c|c|c|}
\hline \multirow{2}{*}{$\begin{array}{l}\text { Componente } \\
\text { de variância }\end{array}$} & \multicolumn{3}{|c|}{ Produção de leite } & \multicolumn{3}{|c|}{ Produção de gordura } \\
\hline & Baixo & Alto & Geral & Baixo & Alto & Geral \\
\hline & \multicolumn{6}{|c|}{ Modelo sem interação } \\
\hline$h^{2}$ & 0,34 & 0,37 & 0,36 & 0,32 & 0,35 & 0,34 \\
\hline $\mathrm{C}^{1}$ & 0,02 & 0,03 & 0,03 & 0,02 & 0,03 & 0,03 \\
\hline \multirow[t]{2}{*}{$\mathrm{e}$} & 0,63 & 0,60 & 0,61 & 0,66 & 0,62 & 0,63 \\
\hline & \multicolumn{6}{|c|}{ Modelo com interação reprodutor $\mathrm{x}$ rebanho } \\
\hline$h^{2}$ & 0,34 & 0,35 & 0,35 & 0,32 & 0,32 & 0,31 \\
\hline $\mathrm{C}^{1}$ & 0,02 & 0,03 & 0,03 & 0,02 & 0,03 & 0,02 \\
\hline $\mathrm{C}^{2}$ & 0,00 & 0,02 & 0,01 & 0,00 & 0,04 & 0,03 \\
\hline \multirow[t]{2}{*}{$\mathrm{e}$} & 0,63 & 0,60 & 0,61 & 0,66 & 0,62 & 0,63 \\
\hline & \multicolumn{6}{|c|}{ Modelo com interação reprodutor x rebanho-ano } \\
\hline$h^{2}$ & 0,34 & 0,36 & 0,36 & 0,32 & 0,35 & 0,34 \\
\hline $\mathrm{C}^{1}$ & 0,02 & 0,03 & 0,03 & 0,02 & 0,03 & 0,03 \\
\hline $\mathrm{C}^{3}$ & 0,02 & 0,02 & 0,03 & 0,02 & 0,01 & 0,03 \\
\hline e & 0,61 & 0,59 & 0,59 & 0,64 & 0,61 & 0,61 \\
\hline
\end{tabular}


As estimativas de herdabilidade foram ligeiramente maiores na classe de alto desviopadrão fenotípico, devido ao aumento da variância genética aditiva em relação ao aumento da variância residual, da classe de baixo para a classe de alto desvio padrão fenotípico. Na classe de baixo desvio-padrão fenotípico, as herdabilidades não se alteraram com a inclusão dos efeitos de interação no modelo, sendo de 0,34 para produção de leite e de 0,32 , para produção de gordura. Na classe de alto desviopadrão fenotípico, as estimativas foram menores nos modelos com interação, sendo de $0,37,0,35$ e 0,36 para produção de leite, e de $0,35,0,32$ e 0,35 , para produção de gordura, nos modelos sem e com interação reprodutor $\mathrm{x}$ rebanho $\mathrm{e}$ reprodutor $\mathrm{x}$ rebanho-ano, respectivamente.

Hill et al. (1983), Dong e Mao (1990) e Araújo (2000), ao estudarem registros de vacas holandesas, encontraram tendência de aumento nas herdabilidades com o aumento no nível de produção dos rebanhos. Segundo Hill et al. (1983), o incremento da estimativa de herdabilidade com a elevação do nível de produção ocorre porque as vacas podem expressar melhor o seu potencial genético em rebanhos com altos níveis de produção, devido ao melhor ambiente oferecido com o controle de enfermidades e a melhor alimentação.

As proporções da variância total referente ao componente de ambiente permanente não diferiram entre as classes de desvio-padrão, e entre os diferentes modelos analisados. A proporção da variância fenotípica para produção de leite, devido à interação reprodutor $\mathrm{x}$ rebanho, foi de 0 e $2,0 \%$, para as classes de baixo e alto desvio-padrão fenotípico, respectivamente, e para interação reprodutor $\mathrm{x}$ rebanho-ano foi de $2,0 \%$ em ambas as classes. Para produção de gordura foi de 0 e $4,0 \%$ com interação reprodutor $\mathrm{x}$ rebanho, e de 2,0 e $1,0 \%$ com interação reprodutor x rebanho-ano, nas classes de baixo e alto desvio-padrão fenotípico, respectivamente.

Araújo (2000), ao estratificar os rebanhos da raça holandesa em três classes (baixo, médio e alto desvio-padrão fenotípico) para produção de leite, verificou que a proporção da variância total, em razão da interação reprodutor x rebanho, variou de 2,6 a 4,4\%, entre as classes de desvios-padrão fenotípicos. Rorato et al. (2000) encontraram valores para as proporções da variância total, devidas à interação touro $\mathrm{x}$ ano-época de, aproximadamente, 7,4 e $4 \%$, respectivamente, para o nível baixo, médio e alto de produção de leite média de rebanho, sugerindo que o efeito pode não ser importante sob o ponto de vista prático.

A proporção de variância total, em razão da variância residual, reduziu da classe de baixo desvio-padrão fenotípico para a classe de alto desvio-padrão fenotípico. Comportamento este, inverso ao apresentado pelas estimativas de herdabilidades. Com isso, presume-se que a maior parte da heterogeneidade de variâncias entre as classes de desvios-padrão fenotípicos, resulte de fatores genéticos. Segundo Garrick e Van Vleck (1987), em situações em que se verificam maiores herdabilidades em ambientes mais variáveis, haveria pequena redução no progresso genético ao se assumir homogeneidade de variâncias.

Costa (1998), ao utilizar registros de produção de leite e de gordura de primeiras lactações de vacas da raça holandesa, os quais foram estratificados com base no desvio-padrão fenotípico das classes de rebanho-ano para produção de leite, nos níveis alto e baixo, verificou aumento dos componentes de variância da classe de baixo para a classe de alto desvio-padrão fenotípico, com maior aumento na variância residual, resultando em estimativas de herdabilidades para as produções de leite e de gordura, maiores $(0,30$ e 0,20, respectivamente) nos rebanhos de baixo desviopadrão, em relação aos de alto desvio-padrão (0,23 e 0,20, respectivamente). Torres (1998), com $o$ intuito de verificar o efeito da heterogeneidade de variâncias na avaliação genética de vacas da raça holandesa no Brasil, dividiu os rebanhos em três classes de desviopadrão fenotípico pela produção de leite, verificando aumento nas médias de produção e nos componentes de variâncias, com o aumento do desvio-padrão médio da classe. As estimativas de herdabilidade não mostraram o mesmo comportamento, sendo maior no nível médio de desvio-padrão $(0,28)$, seguido do nível baixo $(0,26)$ e o menor coeficiente foi encontrado no nível alto $(0,25)$.

Os valores do logaritmo natural da função de verossimilhança e do teste da razão de verossimilhança, utilizados para verificar a significância dos modelos com interação 
reprodutor $\mathrm{x}$ rebanho e reprodutor $\mathrm{x}$ rebanhoano, para as características produção de leite e gordura, em análise geral, e em cada classe de desvio-padrão fenotípico, são apresentados na Tab. 4. Para produção de gordura, a inclusão do efeito de interação reprodutor $\mathrm{x}$ rebanho nos modelos foi significativa, em análise geral e na classe de alto desvio-padrão fenotípico, enquanto $\mathrm{a}$ incorporação da interação reprodutor $\mathrm{x}$ rebanho-ano no modelo foi não significativa para todas as análises. $\mathrm{Na}$ característica produção de leite, não foi observado aumento $(\mathrm{P}<0,01)$ da função de verossimilhança ao considerar os efeitos de interação no modelo.

Tabela 4. Valores do logaritmo natural das funções de verossimilhança $\left(\log _{\mathrm{e}} \mathrm{L}\right)$ e do teste da razão de verossimilhança (LR), para modelos seqüencialmente reduzidos, obtidos para as características produção de leite e gordura, em análises de características única geral e nas classes de desvio-padrão fenotípico

\begin{tabular}{|c|c|c|c|c|c|}
\hline \multicolumn{6}{|c|}{ Produção de leite } \\
\hline & \multicolumn{3}{|c|}{$\log _{\mathrm{e}} \mathrm{L}$} & \multicolumn{2}{|c|}{ LR } \\
\hline $\begin{array}{l}\text { Classe } \\
\text { de desvio- } \\
\text { padrão }\end{array}$ & $\begin{array}{c}\text { Sem } \\
\text { interação }\end{array}$ & $\begin{array}{l}\text { Com interação } \\
\text { reprod. x rebanho }\end{array}$ & $\begin{array}{l}\text { Com interação } \\
\text { reprod. x rebanho- } \\
\text { ano }\end{array}$ & $\begin{array}{c}\text { reprod. } \mathrm{x} \\
\text { rebanho }\end{array}$ & $\begin{array}{l}\text { reprod. } \mathrm{x} \\
\text { reb-ano }\end{array}$ \\
\hline Geral & $-49.528,89$ & $-49.528,35$ & $-49.526,70$ & $1,07^{\mathrm{NS}}$ & $4,37^{\mathrm{NS}}$ \\
\hline Baixo & $-22.414,60$ & $-22.414,60$ & $-22.413,56$ & 0,00 & $2,09^{\mathrm{NS}}$ \\
\hline Alto & $-26.985,06$ & $-26.984,23$ & $-26.984,50$ & $1,66^{\mathrm{NS}}$ & $1,13^{\mathrm{NS}}$ \\
\hline \multicolumn{6}{|c|}{ Produção de gordura } \\
\hline Geral & $-28.383,91$ & $-28.378,61$ & $-28.382,20$ & $10,59^{*}$ & $3,42^{\mathrm{NS}}$ \\
\hline Baixo & $-12.622,59$ & $-12.622,59$ & $-12.621,77$ & 0,00 & $1,64^{\mathrm{NS}}$ \\
\hline Alto & $-15.638,27$ & $-15.634,21$ & $-15.638,04$ & $8,13 *$ & $0,47^{\mathrm{NS}}$ \\
\hline
\end{tabular}

${ }^{\text {NS }}$ não significativo $(\mathrm{P}>0,01)$

$* \mathrm{P}<0,01$

\section{CONCLUSÕES}

A inclusão nos modelos dos efeitos de interação, pouco alteraram as estimativas dos componentes de variâncias, podendo ser desconsiderados na avaliação genética da raça Pardo-Suíça, para produção de leite e gordura, como forma de ajustamento da heterogeneidade de variâncias.

\section{REFERÊNCIAS BIBLIOGRÁFICAS}

ARAÚJO, C.V. de. Efeito da interação reprodutor $x$ rebanho sobre a produção de leite na raça holandesa. 2000. 80f. Dissertação (Mestrado em Zootecnia) - Universidade Federal de Viçosa, Viçosa, MG.

BOLDMAN, K.G.; FREEMAN, A.E. Adjustment for heterogeneity of variance by herd production level in dairy cow and sire evaluation. J. Dairy Sci., v.73, p.503-512, 1990 .

BOLDMAN, K.G.; KRIESE, L.A.; VAN VLECK, L.D. et al. A manual for use of MTDFREML: a set of programs to obtain estimates of variances and covariances (DRAFT). Lincoln: Department of Agriculture / Agriculture Research Service, 1995.

COSTA, C.N. Genetic relationships for milk and fat yields between Brazilian and United States Holstein cattle populations. 1998. 175f. Thesis (Doctor of Philosophy) Cornell University, Ithaca, NY.

DE VEER, J.C.; VAN VLECK, L.D. Genetic parameters for first lactation milk yields at three levels of herd production.
J. Dairy Sci., v.70, p.1434-1441, 1987.

DONG, M.C.; MAO, I.L. Heterogeneity of (co)variance and heritability in different levels of intra-herd milk production variance and of herd average. J. Anim. Sci., v.73, p.843-851, 1990.

GARRICK, D. J.; VAN VLECK, L. D. Aspects of selection for performance in several environments with heterogeneous variances. J. Anim. Sci., v.65, p.409-421, 1987.

HILL, W.G.; EDWARDS, M.R.; AHMED, M.K.A. et al. Heritability of milk yield and composition at different levels and variability of production. Anim. Prod., v.36, p.59-68, 1983.

RAO, C.R. Linear statistical inference and its aplications. New York: John Wiley e Sons, 1973. 552p.

RENNÓ, F.P. Aspectos produtivos da raça Pardo-Suiça no Brasil. 2001. 100f. Dissertação (Mestrado em Zootecnia) Universidade Federal de Viçosa, Viçosa, MG.

RORATO, P.R.N.; VAN VLECK, D.; VERNEQUE, R.S. et al. Interação genótipo-ambiente para a produção de leite em rebanhos da raça holandesa no Brasil. 2. Uso de um modelo animal. Rev. Bras. Zootec., v.29, p.2030-2035, 2000.

TORRES, R.A. Efeito da heterogeneidade de variância na avaliação genética de bovinos da raça Holandesa no Brasil. 1998. 124f. Tese (Doutorado em Ciência Animal) - Escola de Veterinária, Universidade Federal da Minas Gerais, Belo Horizonte, MG.

VINSON, W.E. Potential bias in genetic evaluations from differences in variation within herds. J. Dairy Sci., v.70, p.2450-2455, 1987. 